\title{
Genetic diversity of Mycobacterium tuberculosis isolates from Tochigi prefecture, a local region of Japan
}

Fuminori Mizukoshi ${ }^{1}$, Tohru Miyoshi-Akiyama ${ }^{2,3}$, Hiroki Iwai ${ }^{2}$, Takako Suzuki ${ }^{1}$, Reiko Kiritani ${ }^{1}$, Teruo Kirikae ${ }^{2,4^{*}}$ and Keiji Funatogawa ${ }^{1}$

\begin{abstract}
Background: Foreign-born patients with tuberculosis (TB) may introduce globally disseminated isolates of Mycobacterium tuberculosis into large cities in Japan. The risk of dissemination of these isolates into local regions, however, has not been determined. This study analyzed the molecular epidemiology of $M$. tuberculosis isolates obtained from TB patients living in a local region of Japan.

Methods: Whole genome sequences of 169 M. tuberculosis isolates, obtained from 148 Japanese-born and 21 foreign-born patients living in Tochigi, Japan, were analyzed using the Comprehensive analysis server for the Mycobacterium tuberculosis complex (CASTB).

Results: The 169 isolates were clustered into four clades; Lineage 2 (111 isolates 65.7\%), Lineage 4 (43 isolates, 25. 4\%), Lineage 1 (13 isolates, 7.7\%), and Lineage 3 (2 isolates, 1.2\%). Of the 111 isolates belonging to Lineage 2, 79 (71.2\%) were of the atypical Beijing sub-genotype. Of the 13 Lineage 1 isolates, nine (69.2\%) were from foreignborn patients. The isolates belonging to Lineage 4 were further clustered into three clades, two containing isolates shared by both Japanese- and foreign-born patients. The two isolates belonging to Lineage 3 were obtained from foreign-born patients.
\end{abstract}

Conclusions: The genotypic diversity of $M$. tuberculosis in a local region of Japan is increased primarily by the presence of isolates obtained from foreign-born patients.

Keywords: Foreign-born, Lineage, Whole genome sequencing, CASTB, Japan

\section{Background}

The World Health Organization has estimated that, in 2014, 9.6 million people worldwide were newly infected with tuberculosis $(\mathrm{TB})$ and that 1.5 million people died of this disease [1]. Most Asian countries, except for Japan, the Republic of Korea and Singapore, have been categorized as countries with a high or relatively high TB burden, defined as $\geq 100$ per 100,000 population [1]. The prevalence of TB in large Japanese cities, such as Tokyo, is more than twice that of rural areas [2], with the higher prevalence likely due to the greater presence

\footnotetext{
* Correspondence: tkirikae@ri.ncgm.go.jp; t-kirikae@juntendo.ac.jp

${ }^{2}$ Department of Infectious Diseases, Research Institute, National Center for Global Health and Medicine, Tokyo, Japan

${ }^{4}$ Present Address: Department of Microbiology, Juntendo University School of Medicine, Tokyo, Japan

Full list of author information is available at the end of the article
}

of foreign-born TB patients in large cities. These foreign-born individuals may carry strains of $M$. tuberculosis differing from those present in Japan, reflecting the global diversity of this bacterial species [3]. These patients may therefore introduce globally disseminated isolates of M. tuberculosis into Japan, especially strains from countries with high TB burdens [3]. Less is known, however, about risk factors for $\mathrm{TB}$ in local regions throughout Japan.

Recent molecular epidemiologic studies of $M$. tuberculosis have provided important information on epidemic and drug-resistant strains and on their relationships [4, 5]. M. tuberculosis strains have evolved and become distributed globally during human migrations in response to historical events including the industrial revolution and the two World Wars [5]. Epidemiological analytical 
methods used to assess TB strains have included determination of variable numbers of tandem repeats, spoligotyping, IS6110-based restriction fragment length polymorphism (RFLP) typing, lineage analysis based on long sequence polymorphisms (LSP), and Beijing typing [6-10]. In addition, phylogenetic analysis based on single nucleotide polymorphism (SNP) concatemers is becoming a powerful tool for higher resolution analysis in the era of next-generation sequencing [11-13]. Detection of mutations associated with drug resistance is also important due to the increasing number of patients with drug-resistant TB [14].

The comprehensive analysis server for the Mycobacterium tuberculosis complex (CASTB) is a recently developed web server (http://castb.ri.ncgm.go.jp/CASTB/) [15] that can analyze whole-genome sequencing (WGS) data generated by next-generation sequencers (NGS). Analysis using this server can automatically generate conventional epidemiological results, predict drug resistance, and determine phylogeny based on SNP concatemers. Phylogenetic analysis by CASTB based on SNP concatemers allows high-resolution analysis of relationships among $M$. tuberculosis isolates. CASTB can determine genetic lineages from WGS data, especially from virtual LSP typing. LSP analysis has been used to classify $M$. tuberculosis complexes into six major lineages. Moreover, CASTB can distinguish between isolates belonging to "typical" and "atypical" Beijing sub-lineages, as well as detecting mutations associated with drug resistance and predicting the drug susceptibility of strains.

Tochigi Prefecture of Japan is located $100 \mathrm{~km}$ north of Tokyo, in the center of Honshu, the main island of Japan. This prefecture, which consists of three regions, classified as the northern, central and southern regions, has an area of $6,400 \mathrm{~km}^{2}$ and a population of 2 million people (Additional file 1: Figure S1). To assess the molecular epidemiology of $M$. tuberculosis in a localized area, this study used CASTB to analyze whole genome sequences of $M$. tuberculosis clinical isolates in Tochigi prefecture.

\section{Methods}

\section{Patients and clinical isolates}

A total of 169 clinical isolates of $M$. tuberculosis were obtained from 169 TB patients residing in Tochigi Prefecture. In Japan, the Law Concerning the Prevention of Infections and Medical Care for Patients of Infections mandates that local public health institutions, such as the Tochigi Prefectural Institute of Public Health and Environmental Science (Tochigi IPH), collect all $M$. tuberculosis isolates obtained from TB patients, as well as anonymized clinical information about these patients. The Tochigi IPH began to collect $M$. tuberculosis clinical isolates in 2007. Isolates obtained in 2007 and in 2013 were therefore analyzed in this study. Of the 169 patients, 31, including one foreign-born patient, were living in the northern part of Tochigi; 83, including 13 foreignborn patients, were living in the central part; and 55, including seven foreign-born patients, were living in the southern part (Additional file 2: Table S1). Of these isolates, 87 and 82 were isolated in 2007 and 2013, respectively (Table 1). Of the 87 isolates obtained in 2007, $11(12.6 \%)$ were from foreign-born patients (Table 1) and $60(69.0 \%)$ were from males (Additional file 3: Table S2). Of the 82 isolates obtained in 2013, 10 (12.2\%) were from foreign-born patients (Table 1) and 44 (53.7\%) were from males (Additional file 3: Table S2). The average age of the 169 patients was 62.7 years (range, 1.798.5 years), 37.9 years for patients with $M$. tuberculosis Lineage 1; 69.4 and 57.1 years for patients with Ancestral and Modern Lineage 2, respectively; 35.1 years for patients with Lineage 3; and 63.3 years for patients with Lineage 4 (Fig. 4). The 21 foreign-born patients (Table 1, parentheses) were from nine countries/regions, including four each from Taiwan and the Philippines; three from Korea; two each from China, Nepal, Peru, and Thailand; and one each from Brazil and Vietnam. Information about patients and isolates, including dates and countries/regions of birth, gender, areas of residence, and results of drug susceptibility testing, was obtained from the National Epidemiological Surveillance of Infectious Diseases (NESID), an active infectious disease surveillance system in Japan that collects reports about patients with various infectious diseases [16].

\section{DNA preparation and whole genome sequencing of $\mathrm{M}$. tuberculosis isolates}

Genomic DNA was extracted from all isolates by standard procedures [14]. Paired-end multiplexed Illumina sequencing was performed using MiSeq (Illumina Inc., San Diego, CA, USA), followed by mapping of sequence reads [17].

Table 1 Lineage distribution of M. tuberculosis isolates in 2007 and 2013

\begin{tabular}{|c|c|c|c|c|c|c|}
\hline & \multicolumn{6}{|c|}{ Lineage/Beijing typing } \\
\hline & \multirow[t]{2}{*}{ Lineage 1} & \multicolumn{2}{|l|}{ Lineage 2} & \multirow[t]{2}{*}{ Lineage 3} & \multirow[t]{2}{*}{ Lineage 4} & \multirow[t]{2}{*}{ Total } \\
\hline & & $\begin{array}{l}\text { Ancestral } \\
\text { (Atypical) }\end{array}$ & $\begin{array}{l}\text { Modern } \\
\text { (Typical) }\end{array}$ & & & \\
\hline \multirow[t]{2}{*}{ Total } & $13(9)$ & $111(4)$ & & $2(2)$ & $43(6)$ & $169(21)$ \\
\hline & & 79 (3) & $32(1)$ & & & \\
\hline 2007 & $\begin{array}{l}8(5) \\
{[9.2 \%]}\end{array}$ & $\begin{array}{l}44(3) \\
{[50.6 \%]}\end{array}$ & $\begin{array}{l}13(0) \\
{[14.9 \%]}\end{array}$ & $\begin{array}{l}0(0) \\
{[0.0 \%]}\end{array}$ & $\begin{array}{l}22(3) \\
{[25.3 \%]}\end{array}$ & $\begin{array}{l}87(11) \\
{[100.0 \%]}\end{array}$ \\
\hline 2013 & $\begin{array}{l}5(4) \\
{[6.1 \%]}\end{array}$ & $\begin{array}{l}35(0) \\
{[42.7 \%]}\end{array}$ & $\begin{array}{l}19(1) \\
{[23.2 \%]}\end{array}$ & $\begin{array}{l}2(2) \\
{[2.4 \%]}\end{array}$ & $\begin{array}{l}21(3) \\
{[25.6 \%]}\end{array}$ & $\begin{array}{l}82(10) \\
{[100.0 \%]}\end{array}$ \\
\hline
\end{tabular}

Numbers in parentheses represent numbers of foreign-born TB patients Numbers in brackets represent percentages of foreign-born TB patients 


\section{CASTB analysis}

WGS data, generated by NGS, were analyzed using the CASTB web server [15]. Conventional epidemiological results, predicted resistance against drugs (ciprofloxacin, ethambutol, pyrazinamide, rifampin and streptomycin), and lineage analysis based on virtual LSP and SNP concatemers, as well as spoligotyping, were automatically obtained from sequence data.

\section{Phylogenetic analyses using maximum likelihood (ML) and neighbor-joining (NJ) methods}

The ML and NJ methods were used for phylogenetic analyses. The identified SNPs were concatenated to obtain concatenated SNP sequences for alignment with MAFFT [18]. After obtaining the best model for the ML tree, a phylogenetic tree was generated by aligning SNPs using a general time reversible model, with MEGA version 7, PhyML3.0 [19] and FigTree v1.4.0. software (http://tree.bio.ed.ac.uk/software/figtree/).

\section{Statistical analyses}

Data were summarized as mean, median, and/or range, as appropriate, and compared using Fisher's exact test or the nonparametric Student's $t$ test. All tests were two-sided, with $p<0.05$ considered statistically significant.

\section{Results}

\section{Phylogenetic and lineage analyses}

ML trees were constructed from the 169 M. tuberculosis isolates obtained from patients registered in the NESID in 2007 and 2013 (Fig. 1a and b). These ML trees were congruent with the trees constructed by the neighborjoining method for each of these years (Fig. 2a and b). Phylogenetic analysis revealed four clades in good agreement with four of the lineages (Lineages 1-4) categorized by genotyping based on LSP (Figs. 1 and 2). Of the 169 isolates, 111 (65.7\%) belonged to Lineage 2 (East Asian or Beijing), 43 (25.4\%) to Lineage 4 (Euro-American), 13 (7.7\%) to Lineage 1 (Indo-Oceanic), and two (1.2\%) to Lineage 3 (East African-Indian) (Table 1). Of the 111 isolates belonging to Lineage 2, 32 (28.8\%) belonged to the typical (modern) Beijing sub-genotype, and 79 (71.2\%) to the atypical (ancestral) Beijing sub-genotype. Fifty-seven (65.5\%) of the 87 isolates obtained in 2007, and 54 (65.9\%) of the 82 isolated in 2013 belonged to Lineage 2, with 44 (50.6\%) and 35 (42.7\%), respectively, belonging to the atypical Beijing sub-genotype (Table 1). The percentage of isolates belonging to the typical Beijing sub-genotype was higher in 2013 (19/82, 23.2\%) than in 2007 (13/87, 14.9\%).

Three ML trees were constructed from isolates belonging to Lineages 1, 2 and 4, respectively (Fig. 3). The 13 isolates belonging to Lineage 1 did not form any subclades (Fig. 3a). The 111 isolates belonging to Lineage 2 could be divided into four sub-clades, containing 26, 22, 31 and 32 isolates, respectively (Fig. 3b). Isolates belonging to clades 1, 2 and 3 were of the atypical (ancestral) Beijing sub-genotype, with the isolates belonging to subclade 4 being of the typical Beijing sub-genotype (Fig. 3b). Forty-three isolates belonged to Lineage 4 (Fig. 3c). Both isolates belonging to Lineage 3 were detected in 2013 (Fig.1a), with none detected in 2007 (Fig. 1b).

\section{Lineage comparison Isolates from foreign- and Japanese-born patients}

Of the 169 isolates, 21 (12.4\%) were from foreign-born patients, with nine, four, two, and six belonging to Lineages 1,2 , Lineage 3 , and 4, respectively (Table 1 ). Of the 13 Lineage 1 isolates nine (69.2\%) were from foreignborn patients (Table 1 and Fig. 3a), including three patients from the Philippines, two each from Taiwan and Thailand, and one each from Nepal and Vietnam. Of the 111 isolates belonging to Lineage 2, 107 (96.4\%) were from Japan-born patients and only four (3.6\%) from foreign-born patients (Table 1), with one each of the latter belonging to each of the four sub-clades of Lineage 2 (Fig. 3b). One isolate, belonging to the typical subgenotype of Lineage 2, was obtained from a patient born in China, whereas the other three were from patients born in Korea, Taiwan, and China, respectively. The two Lineage 3 isolates detected in 2013 (Table 1) were obtained from foreign-born patients, a man in his twenties from Nepal and a woman in her forties from the Philippines, both of whom were living in the southern region of Tochigi. Of the 43 isolates belonging to Lineage 4, six (14.0\%) were from foreign-born patients, including two each from Korea and Peru and one each from Brazil and Thailand (Fig. 3c).

\section{Elderly and nonelderly patients}

Figure 4 shows the age distribution from which isolates of each lineage were obtained. Among patients infected with Lineage 2 isolates, those infected with Ancestral strains were significantly older than those infected with Modern strains $(p=0.0095)$. Of the 79 patients with Lineage 2-Ancestral isolates and the 22 with Lineage 4 isolates in 2007 (Table 1), 78 and 20, respectively developed TB at the indicated ages (Fig. 4). Japan-born patients with Lineage 1 isolates were significantly younger than patients with Lineage 2 -Ancestral $(p=1.15 \mathrm{E}$ $06)$, Lineage 2 -Modern $(p=0.0016)$, and Lineage 4 $(p=4.21 \mathrm{E}-05)$ isolates. Patients with isolates belonging to Lineage 1, both Japan- and foreign-born, were also significantly younger than either Japan-born patients with Lineage 2-Ancestral $(p=9.38 \mathrm{E}-04)$ or Lineage $4(p=1.56 \mathrm{E}-03)$ isolates. Both patients with 


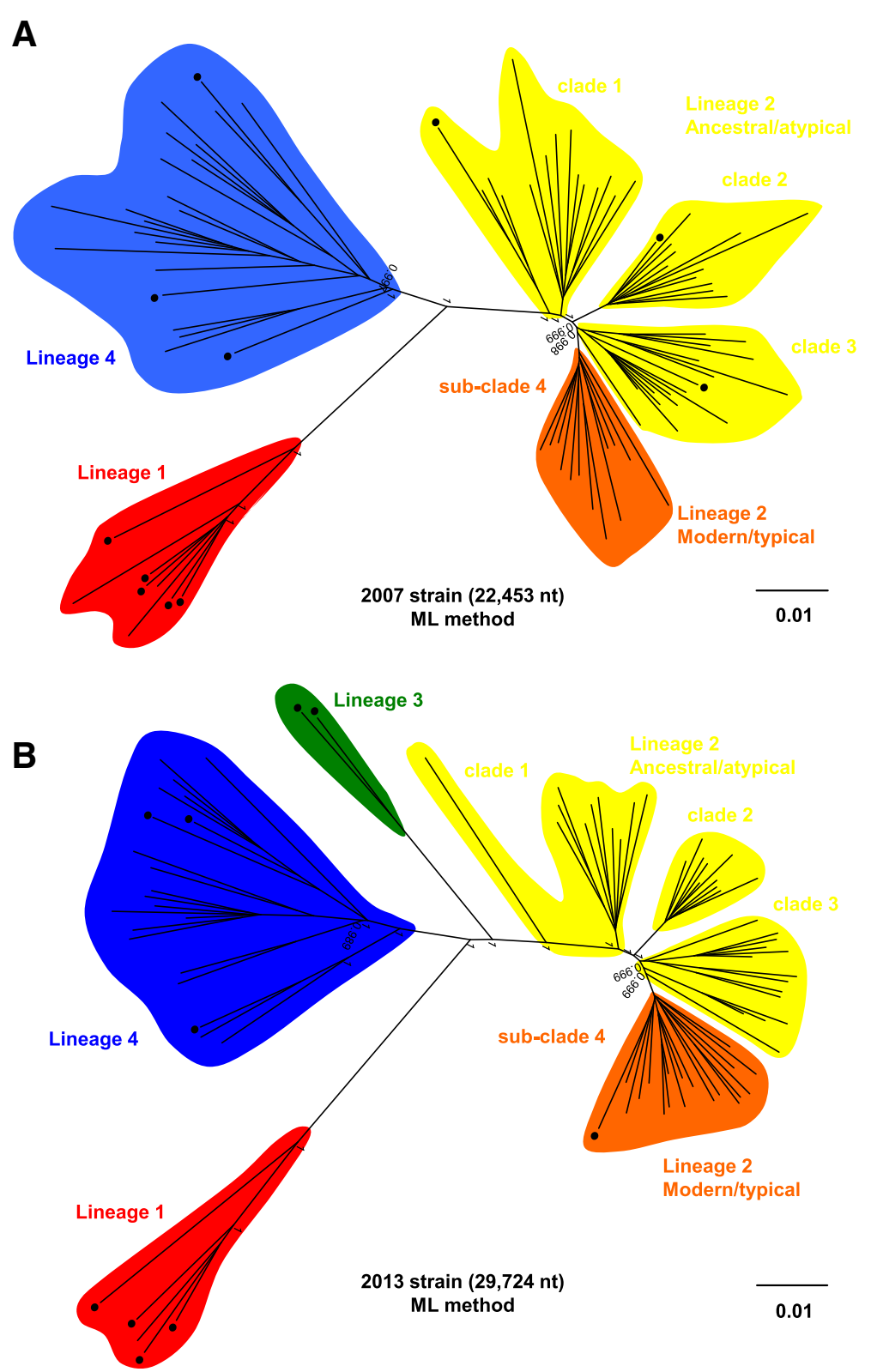

Fig. $1 \mathrm{ML}$ phylogenetic trees based on SNP concatenated sequence alignments of clinical isolates. ML phylogenetic trees of 169 clinical isolates based on whole genome analysis. Radial rectangular tree layouts are shown for the years of M. tuberculosis isolation, a 2007 and b 2013. Bootstrap values are shown in the phylogenetic trees. Clades show Lineage 1 (red), Lineage 2 (orange [Modern/typical] and yellow [Ancestral/atypical]), Lineage 3 (green) and Lineage 4 (blue). Scale bars indicate nucleotide substitutions per site. Black circles indicate foreign-born TB patients

Lineage 3 isolates were relatively young, one in his twenties and the other in her forties.

\section{Residential regions of patients (northern, central and southern parts of Tochigi prefecture)}

Both patients infected with Lineage 3 isolates lived in the southern part of Tochigi prefecture. There were no other relationships between the Lineages of isolates and residential regions (Additional file 4: Figure S2 and Additional file 2: Table S1).

\section{Patient gender}

There were twice as many male as female patients in 2007, with the numbers of males infected with Lineage 1,2 , and 4 isolates in that year being higher than those of females (Additional file 3: Table S2). The number of 

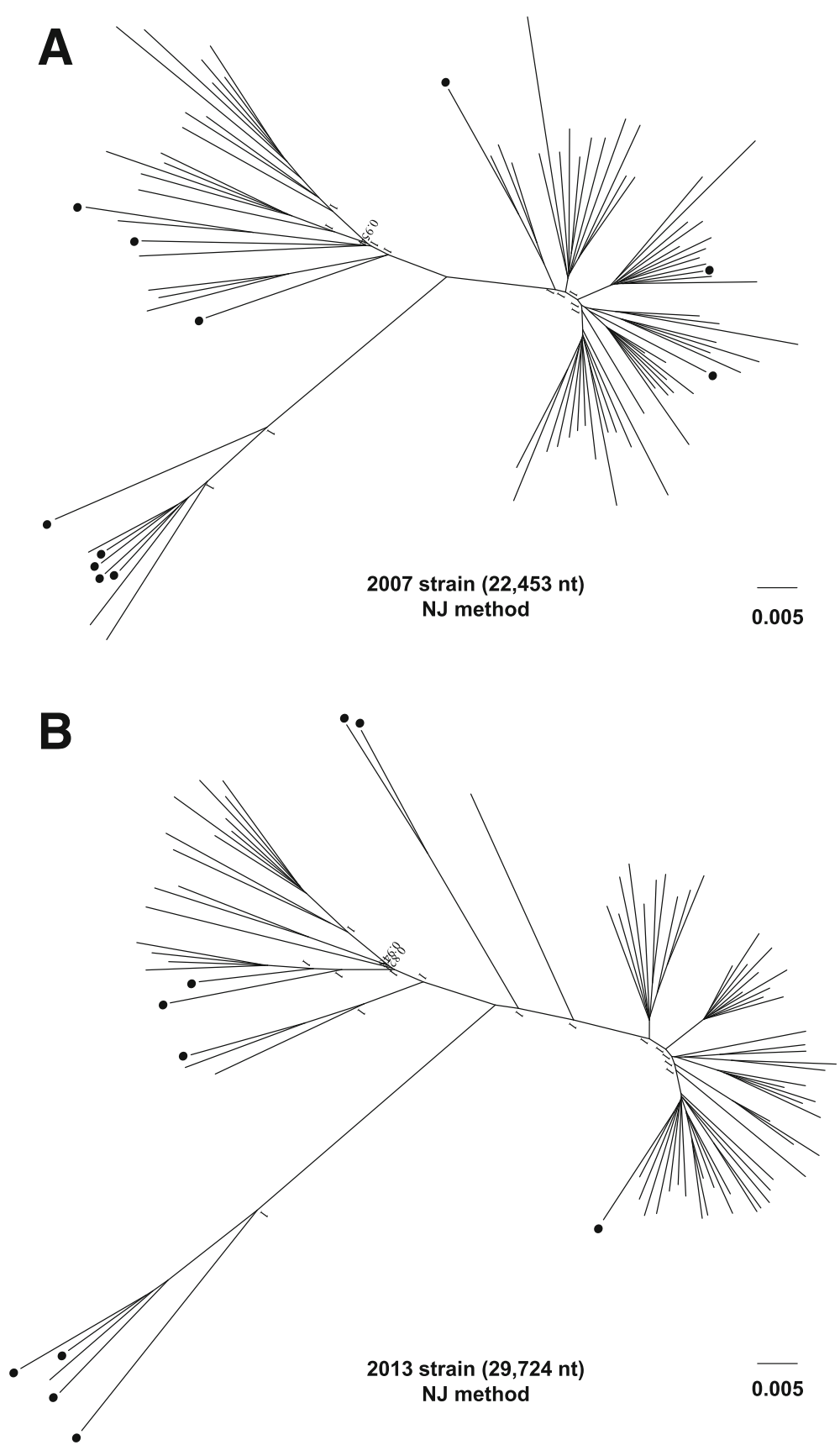

Fig. 2 NJ phylogenetic trees based on SNP concatenated sequence alignments of clinical isolates. NJ phylogenetic trees of the 169 clinical isolates based on whole genome analysis. Radial rectangular tree layouts are shown for the years of M. tuberculosis isolation, a 2007 and b 2013 . Bootstrap values are shown in the phylogenetic trees. Scale bars indicate nucleotide substitutions per site. Black circles indicate foreign-born TB patients

male patients was slightly higher than the number of females in 2013 (Additional file 3: Table S2).

\section{Drug susceptibility}

CASTB analysis showed that, of the 169 isolates, 11 (6.5\%) were resistant to at least one drug, including five resistant to isoniazid, three resistant to streptomycin, and one each resistant to ciprofloxacin, isoniazid + streptomycin, and isoniazid
+ streptomycin + ciprofloxacin. Of the five isoniazid-resistant isolates, three belonged to Lineage 1 and two to Lineage 4 . The three isolates belonging to Lineage 1 were from three patients born in the Philippines, whereas the two belonging to Lineage 4 were from two Japan-born patients. One isolate resistant to both isoniazid and streptomycin belonged to Lineage 2 and was from a patient born in China. The isolate resistant to ciprofloxacin belonged to Lineage 4 and was 


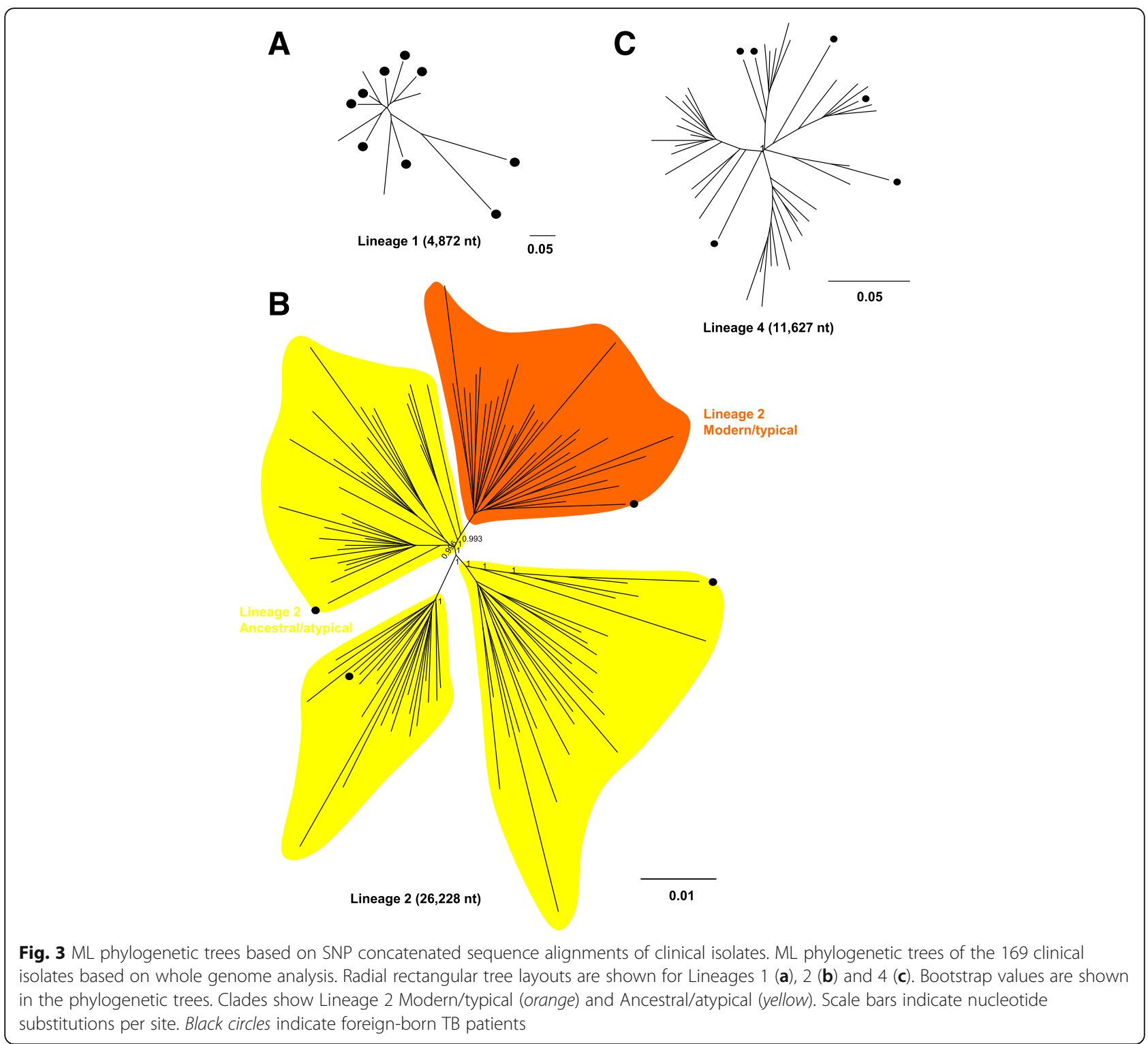

from a Japan-born patient. One of the isolates resistant to isoniazid, streptomycin and ciprofloxacin belonged to Lineage 2 and was from a Japan-born patient. Collectively, of the 11 drug-resistant isolates, four were from foreign-born patients, with the percentage of drug-resistant isolates being significantly higher in foreign-born than in Japan-born patients $(p=0.033)$.

NESID reports included the results of drug susceptibility tests for $86(50.9 \%)$ of 169 isolates, not for the other 83. Of the 86 isolates, three were resistant to at least one drug, with one each resistant to isoniazid, isoniazid + ethionamide, and isoniazid + streptomycin + levofloxacin. The drug susceptibility profiles of these isolates essentially agreed with those of CASTB analysis (Additional file 5: Table S3).

\section{Discussion}

This study clearly showed that foreign-born TB patients affect the genetic diversity of $M$. tuberculosis isolates in Tochigi Prefecture, a local region of Japan. This conclusion was supported by results showing that most of the Lineage 1 isolates were from foreign-born patients, as were both of the Lineage 3 isolates. We previously reported that foreign-born TB patients also affect the genetic diversity of isolates in the center of metropolitan Tokyo [3]. These results indicate that foreign-born TB patients have an impact on the nationwide genetic diversity of M. tuberculosis in Japan.

TB epidemiology has been analyzed in foreign-born patients living in countries with low TB prevalence, including Madrid and Catalonia, Spain [20], the USA 


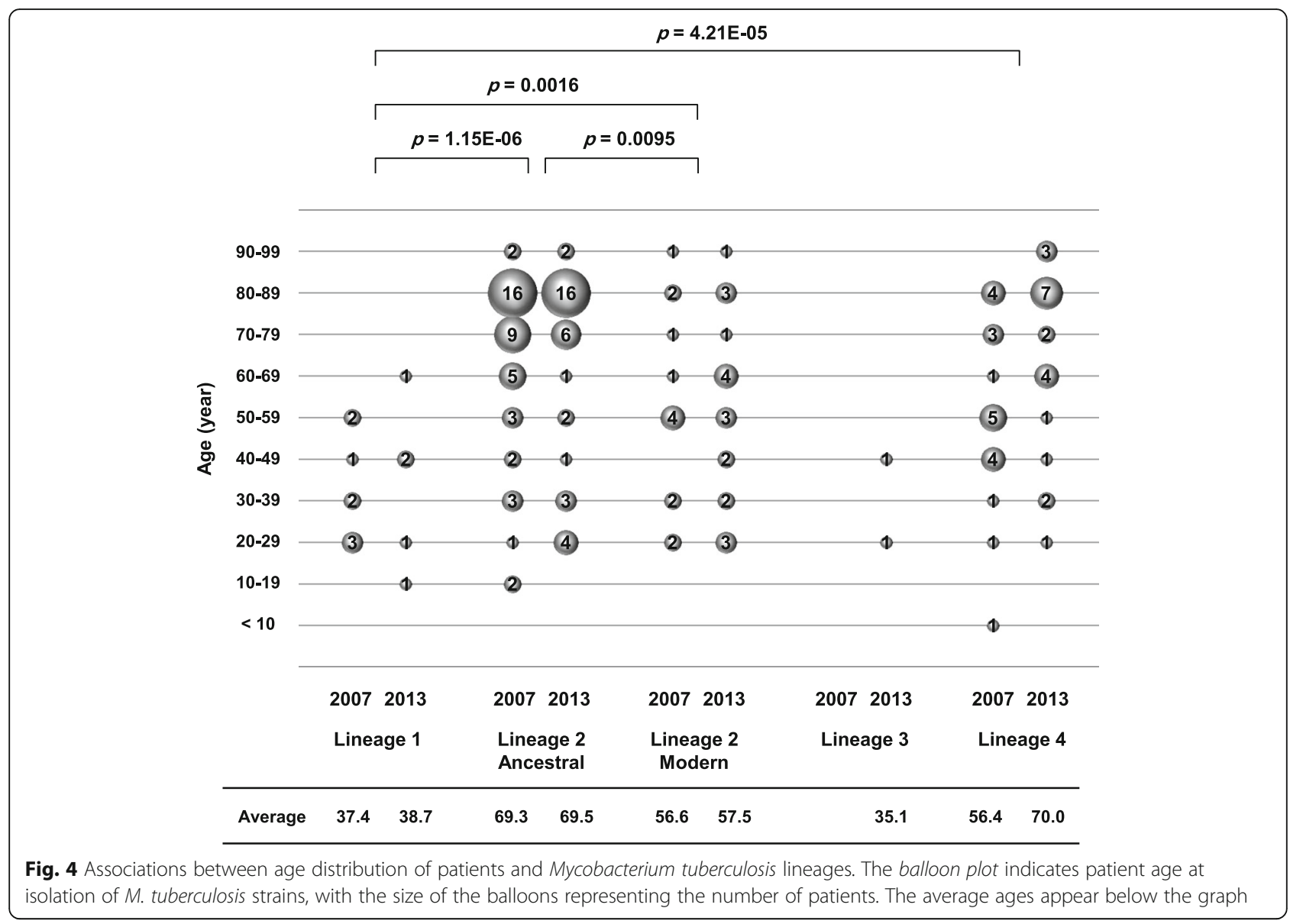

(http://www.ingentaconnect.com/), Canada [21], Switzerland [22], and Germany [23]. Although most of these studies found little transmission of $M$. tuberculosis from foreignborn patients to native-born residents, our phylogenetic and lineage analyses predicted $M$. tuberculosis transmission among foreign-born and Japan-born TB patients. The difference in the impact of foreign-born $\mathrm{TB}$ patients on $\mathrm{TB}$ epidemiology may be due to the prevalence of TB being higher in Japan, a country with a middle TB-burden, than in developed countries, with a low TB-burden, as well as to differences in employment and lifestyles.

These findings also showed the globalization of $M$. tuberculosis transmission in a local region of Japan. TB isolates from foreign-born patients may increase TB genetic diversity in Tochigi Prefecture. Although no direct evidence has shown that these isolates from foreign-born patients in Tochigi are a risk factor for TB transmission, similar findings were reported for TB isolates from foreign-born and Japan-born TB patients in Tokyo [3]. Of the 13 isolates belonging to Lineage 1 , nine were from patients born in other Asian countries, including seven from Southeast Asian countries/regions, where Lineage 1 is predominant $[6,24]$. In addition, three of the 43 Lineage 4 isolates were from patients born in South America, where
Lineage 4 is predominant $[6,24]$. Of individuals living in Tochigi in 2007 and 2013, only $1.7 \%$ and 1.5\%, respectively, were registered foreigners (http://www.pref.tochigi.lg.jp/ f04/documents/h27jumin-sityoubetu.pdf; the Tochigi prefectural websites in Japanese), whereas $12.6 \%$ and $12.2 \%$, respectively, of isolates obtained nationwide during these 2 years were from foreign-born patients. Beginning in 2012, more than 1,000 foreign-born TB patients are newly registered annually in Japan, with the numbers continuing to increase (http://www.mhlw.go.jp/bunya/kenkou/kekkakukansenshou03/14.html; in Japanese). The globalization and diversity of $M$. tuberculosis are increasing in Japan, making molecular epidemiology studies of TB isolates obtained from both foreign-born and native-born patients necessary.

\section{Conclusion}

In conclusion, whole genome analysis of $169 \mathrm{M}$. tuberculosis isolates in Tochigi prefecture using CASTB revealed the genetic diversity of $M$. tuberculosis isolates in a local area of Japan, as well as the impact of foreign-born TB patients. Isolates from foreign-born TB patients were apparently not transmitted extensively to Japan-born residents, these foreign-born 
patients carry a broader lineage of TB strains. CASTB is a useful tool for assessing the epidemiology of $M$. tuberculosis.

\section{Additional files}

Additional file 1: Figure S1. Geographical location of Tochigi Prefecture in Japan. Tochigi is one of the inland prefectures of the Northern portion of the Kanto region. Its population on March 1, 2007, was 2,014,931 persons. The area of Tochigi prefecture is approximately $6,400 \mathrm{~km}^{2}$, making it the 20th largest in Japan, but the largest in the Kanto region. (PPTX $127 \mathrm{~kb}$ )

Additional file 2: Table S1. Geographic analysis of lineages of $M$. tuberculosis isolates obtained from foreign- and Japanese-born patients living in the North, Central, and South regions of Tochigi Prefecture. (DOCX $31 \mathrm{~kb})$

Additional file 3: Table S2. Associations between patient gender and lineages of $M$. tuberculosis isolates obtained from foreign- and Japaneseborn patients. (DOCX $31 \mathrm{~kb})$

Additional file 4: Figure S2. Associations between patient gender and Mycobacterium tuberculosis lineages. The stacked bar charts show the percentages of $M$. tuberculosis lineages by $(A)$ countries of birth and (B) sampling period. The numbers of isolates are indicated on the graphs. (PPTX $133 \mathrm{~kb}$ )

Additional file 5: Table S3. Drug susceptibility and resistance of $M$. tuberculosis isolates. (DOCX $31 \mathrm{~kb}$ )

\section{Abbreviations}

CASTB: The comprehensive analysis server for the Mycobacterium tuberculosis complex; LSP: Lineage analysis based on long sequence polymorphisms; ML: Maximum likelihood; NESID: National epidemiological surveillance of infectious diseases; NGS: Next-generation sequencers; NJ: Neighbor-joining; SNP: Single nucleotide polymorphism; TB: Tuberculosis; WGS: Whole-genome sequencing

\section{Acknowledgements}

The authors wish to thank the patients and staff at both facilities.

\section{Funding}

This study was supported by Grants for International Health Research (27A1102, T.K.; 26A-103, T.M.A.) from the Ministry of Health, Labor, and Welfare of Japan, and was (partially) supported by the Research Program on Emerging and Re-emerging Infectious Diseases from Japan Agency for Medical Research and development, AMED (Research Program on Emerging and Re-emerging Infectious Diseases; 16fk0108302h0003).

\section{Availability of data and materials}

Data generated in this study will not be made available to protect the confidentiality of participants.

\section{Authors' contributions}

Conceived and designed the experiments: TK, TMA, KF. Performed the experiments: FM. Analyzed the data: FM, TMA. Wrote, read and approved the manuscript FM, TMA, HI, TS, RK, TK, KF.

\section{Competing interests}

The authors declare that they have no competing interests.

\section{Consent for publication}

Not applicable.

\section{Ethics approval and consent to participate}

Ethical approval without patient informed consent for this de-identified retrospective medical record review was obtained from Ethical Committee of Tochigi Prefectural Institute of Public Health and Environmental Science (approval numbers :H27-131629 and H27-143891) and National Center for Global Health and Medicine (approval number: NCGM-G-001467-00).

\section{Publisher's Note}

Springer Nature remains neutral with regard to jurisdictional claims in published maps and institutional affiliations.

\section{Author details}

${ }^{1}$ Tochigi Prefectural Institute of Public Health and Environmental Science, Utsunomiya, Tochigi, Japan. ${ }^{2}$ Department of Infectious Diseases, Research Institute, National Center for Global Health and Medicine, Tokyo, Japan. ${ }^{3}$ Pathogenic Microbe Laboratory, Research Institute, National Center for Global Health and Medicine, Tokyo, Japan. ${ }^{4}$ Present Address: Department of Microbiology, Juntendo University School of Medicine, Tokyo, Japan.

Received: 7 November 2016 Accepted: 9 May 2017

Published online: 25 May 2017

References

1. World Health Organization. Global tuberculosis report 2015. Geneva: World Health Organization; 2015

2. Estimates of National Medical Care Expenditure 2013. Available: http://www. mhlw.go.jp/english/wp/wp-hw9/dl/02e.pdf

3. Kato-Miyazawa M, Miyoshi-Akiyama T, Kanno Y, Takasaki J, Kirikae T, Kobayashi N. Genetic diversity of Mycobacterium tuberculosis isolates from foreign-born and Japan-born residents in Tokyo. Clin Microbiol Infect. 2015;21(3):248.e1-8.

4. Comas I, Coscolla M, Luo T, Borrell S, Holt KE, Kato-Maeda M, et al. Out-ofAfrica migration and Neolithic coexpansion of Mycobacterium tuberculosis with modern humans. Nat Genet. 2013:45:1176-82.

5. Gagneux S, Small PM. Global phylogeography of Mycobacterium tuberculosis and implications for tuberculosis product development. Lancet Infect Dis. 2007;7:328-37.

6. Gagneux S, DeRiemer K, Van T, Kato-Maeda M, de Jong BC, Narayanan S, et al. Variable host-pathogen compatibility in Mycobacterium tuberculosis. Proc Natl Acad Sci U S A. 2006;103:2869-73.

7. Mathema B, Kurepina NE, Bifani PJ, Kreiswirth BN. Molecular epidemiology of tuberculosis: current insights. Clin Microbiol Rev. 2006;19:658-85.

8. Mazars E, Lesjean S, Banuls AL, Gilbert M, Vincent V, Gicquel B, et al. Highresolution minisatellite-based typing as a portable approach to global analysis of Mycobacterium tuberculosis molecular epidemiology. Proc Natl Acad Sci U S A. 2001;98:1901-6.

9. Thierry D, Brisson-Noel A, Vincent-Levy-Frebault V, Nguyen S, Guesdon JL Gicquel B. Characterization of a Mycobacterium tuberculosis insertion sequence, IS6110, and its application in diagnosis. J Clin Microbiol. 1990;28: 2668-73.

10. Hirsh AE, Tsolaki AG, DeRiemer K, Feldman MW, Small PM. Stable association between strains of Mycobacterium tuberculosis and their human host populations. Proc Natl Acad Sci U S A. 2004;101:4871-6.

11. Okumura K, Kato M, Kirikae T, Kayano M, Miyoshi-Akiyama T. Construction of a virtual Mycobacterium tuberculosis consensus genome and its application to data from a next generation sequencer. BMC Genomics. 2015;16:218. 015-1368-9.

12. Sekizuka T, Yamashita A, Murase Y, Iwamoto T, Mitarai S, Kato S, et al. TGSTB: total genotyping solution for Mycobacterium tuberculosis using shortread whole-genome sequencing. PLoS One. 2015;10, e0142951.

13. Walker TM, Kohl TA, Omar SV, Hedge J, Del Ojo EC, Bradley P, et al. Wholegenome sequencing for prediction of Mycobacterium tuberculosis drug susceptibility and resistance: a retrospective cohort study. Lancet Infect Dis. 2015;15:1193-202

14. Sekiguchi J, Nakamura T, Miyoshi-Akiyama T, Kirikae F, Kobayashi I, Augustynowicz-Kopec E, et al. Development and evaluation of a line probe assay for rapid identification of pncA mutations in pyrazinamide-resistant Mycobacterium tuberculosis strains. J Clin Microbiol. 2007:45:2802-7.

15. Iwai $\mathrm{H}$, Kato-Miyazawa M, Kirikae T, Miyoshi-Akiyama T. CASTB (the comprehensive analysis server for the Mycobacterium tuberculosis complex): A publicly accessible web server for epidemiological analyses, drugresistance prediction and phylogenetic comparison of clinical isolates. Tuberculosis (Edinb). 2015;95:843-4

16. Infectious Disease Surveillance Center. Sentinel Surveillance [in Japanese]. Tokyo: Ministry of Health, Labour, and Welfare, National Institute of Infectious Diseases; 2010. Available: http://idsc.nih.go.jp/idwr/index-e.html.

17. Miyoshi-Akiyama T, Takeshita N, Ohmagari N, Kirikae T. Complete genome sequence of Helicobacter cinaedi type strain ATCC BAA-847. J Bacteriol. 2012 194:5692. 
18. Katoh K, Misawa K, Kuma K, Miyata T. MAFFT: a novel method for rapid multiple sequence alignment based on fast Fourier transform. Nucleic Acids Res. 2002;30:3059-66.

19. Guindon S, Dufayard JF, Lefort V, Anisimova M, Hordijk W, Gascuel O. New algorithms and methods to estimate maximum-likelihood phylogenies: assessing the performance of PhyML 3.0. Syst Biol. 2010;59:307-21.

20. Inigo J, Garcia de Viedma D, Arce A, Palenque E, Alonso Rodriguez N,

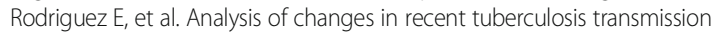
patterns after a sharp increase in immigration. J Clin Microbiol. 2007;45:63-9.

21. Kunimoto D, Sutherland K, Wooldrage K, Fanning A, Chui L, Manfreda J, et al. Transmission characteristics of tuberculosis in the foreign-born and the Canadian-born populations of Alberta, Canada. Int J Tuberc Lung Dis. 2004; 8:1213-20.

22. Fenner L, Gagneux S, Helbling P, Battegay M, Rieder HL, Pfyffer GE, et al. Mycobacterium tuberculosis transmission in a country with low tuberculosis incidence: role of immigration and HIV infection. J Clin Microbiol. 2012;50: 388-95.

23. Barniol J, Niemann S, Louis VR, Brodhun B, Dreweck C, Richter E, et al. Transmission dynamics of pulmonary tuberculosis between autochthonous and immigrant sub-populations. BMC Infect Dis. 2009;9:197. 2334-9-197.

24. Reed MB, Pichler VK, Mclntosh F, Mattia A, Fallow A, Masala S, et al. Major Mycobacterium tuberculosis lineages associate with patient country of origin. J Clin Microbiol. 2009;47:1119-28.

\section{Submit your next manuscript to BioMed Central and we will help you at every step:}

- We accept pre-submission inquiries

- Our selector tool helps you to find the most relevant journal

- We provide round the clock customer support

- Convenient online submission

- Thorough peer review

- Inclusion in PubMed and all major indexing services

- Maximum visibility for your research

Submit your manuscript at www.biomedcentral.com/submit 